Témoigner Témoigner. Entre histoire et mémoire

Getuigen Revue pluridisciplinaire de la Fondation Auschwitz

$118 \mid 2014$

Au nom des victimes. Dictature et terreur d'État en Argentine, Chili et Uruguay

\title{
De la circulation des croix gammées
}

On the Circulation of Swastikas

Overal hakenkruizen

Philippe Mesnard

\section{CpenEdition}

Journals

\section{Édition électronique}

URL : http://journals.openedition.org/temoigner/868

DOI : 10.4000/temoigner.868

ISSN : 2506-6390

Éditeur :

Éditions du Centre d'études et de documentation Mémoire d'Auschwitz, Éditions Kimé

Édition imprimée

Date de publication : 1 octobre 2014

Pagination : 4-5

ISBN : 978-2-84174-674-3

ISSN : 2031-4183

Référence électronique

Philippe Mesnard, «De la circulation des croix gammées », Témoigner. Entre histoire et mémoire [En ligne], 118 | 2014, mis en ligne le 01 octobre 2015, consulté le 23 octobre 2020. URL : http:// journals.openedition.org/temoigner/868 ; DOI : https://doi.org/10.4000/temoigner.868 


\section{DE LA CIRCULATION DES CROIX GAMMÉES} $\rightarrow$ Par Philippe Mesnard Directeur de la rédaction

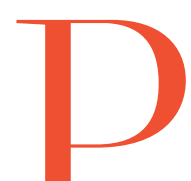

_ rofitons de ce que l'éditorial d'un quadrimestriel est, par obligation, inactuel pour exprime quelques considérations rétrospectives. Il y a moin dun an, les médias póntiniens ressortaient la croix dérive fazciste des Ulrainiens. Phraséols appelaient che disait jadis, visait à disqualifier les orientations inde

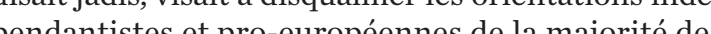
pendantistes et pro-européennes de la majorité des Ianoukovitch, et à légitimer les positions potentielleTher.

Jusque-là, on est d'accord, n'est-ce pas ? MAIS U'UN DANS LUSAGE DUMAUDT SYMBOLE da presse européenne l'a facilement sounten, conme la presse européenne la facilement soutenu, n'est-ce pasfaire porter àl Ukraine les habits trop confortables déjà dire que j'exà̀re, que je ne peux pas. J'entend éja dire que jexagere, que je ne peux pas traiter à la épere la sanglante rép cuind pposants de la place Maidan.

Eas un autre côte, conme disait Perec, les faits ne parlen pas deux-mêmes. Ils ont été digerés, d un côté, dans un la trars pan européen qui avait beau jeu de présenter qualité, de l'autre, par le discours du parti de Poutine Or si lau tine. Or, si les stratagèmes de ce dernier sont faciles à reperrer, on peut cependant sinteresser de plus pres à lá libre circulation du svastika et aux vérités qứelle à l'égerd de l'extrémisme ukrainien. Lacécité d'
Europe dont les dernières élections parlementaire trévéléla force del'extrême droite. À méditer, non?

Mais s'agit-il ici de parler de l’Ukraine (cible de la propaga se en réalité L particulier sert d'exemese Des deux, vientill. Le particulier sert dexenple au genéral, vient inlustrer etle rappeler. Ilest difficile de circule sur ce terrain où ceux qui sontreconnus pour êt pointent des vérités malgré tout C'est pointent des... vérités nalgré tout. Cest sur ce point que je voudrais me prononcer plus précisément. On

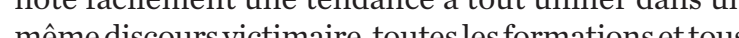
mes gro discoù vin tique. Ace titre, le grand enjeu de la transmission, ce ne serait pas (ou plus) tant linformation - omnipresente, aujourd hui, Jusquá satúation -, que d'apprendre à qu'elle designe partois de façon lointaine alors quelle cus fait accroire quelle traite du reeel « en direct ».

Certes, la complexité est un mot qui peut auss plexité, on doit auss Toutens, aù nom de cette complexité, on doit aussi apprendre à suspendre ces jugemieux discer foce que lon nous de la victime et à mieux discerner ce que lon nous montre. Il faut ains rappeler la permanence du fond ultranationaliste ukrainien qui honore toujours ses hèros pronazis coupables de crimes antisémites, Yaroslav Stetsko et Stepan Bandera, pour ne nommer qu'eux. D'ailleurs la lui élève une de denier a about en 2010 à ce quoon tique ì cetitre legrals sonthach surelle désigne parfois de facon lointaine an désigne. lui eleve une statue, rien de moins! Autrement dit, ce régime autoritaire de Vladimir Poutine, que les dominantes politiques ukrainiennes sont toutes unanimement proches de la démocratie telle qưelle est conçue (imaginée) dans la communauté européenne. D’ailleurs, interdire la langue russe fin février 2014, sitôt constitué le pouvoir intérimaire d'Oleksandr Tourtchynov, ne donnait guère des gages de démocratie. Pour le dire de façon un peu sommaire: les ennemis de nos ennemis ne sont pas nécessairement nos amis.

LA FORMULE PARAÎT CLINQUANTE, IL EST VRAI. Le problème - car il est important de maintenir un d́batene - car il est important de maind'extrémistes urainiens ne sont pas une rason pour disqualifier la société tout entière t ne paison pour disqualifier la société tout entiere et ne pas croire en Il faut rappeler la triste histoire d'une Ukraino tirilÍe, découpée, envahie des sièces du Ukr parne tiraillée, découpée, envahie des siècles durant par ses voisins (Pologne, Autriche, Allemagne, Russie tsariste, Union soviétique). Rappeler les deux grandes famines, la répressionetles déportations connunistes dansles années trente, puis apres-guere. Lourd contentieux l'ex-Union soviétique. Sur cette Bloodland, la gue 'ex-Union soviétique. Sur cette Bloudland, la guerre Et des sauvageries.

DES SAUVAGERIES, EXACTEMENT. Au moins, il n'y en avait plus sur les Juifs car dans ces contrées, de Juifs, il ait plus sur les Juifs car dansces contrées, des Juirs, in n'yé vait plus. llaut dire que leur décination 'ost ane pogite sest amplement poursuivie durant la guerre de $1914-$ 19. véritan cens, la propagande poutinienne pointe semble des pays baltes, n'est jamais vraime quelen-

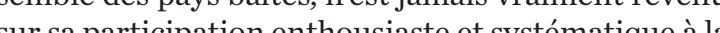
sur sa participation enthouslaste et systematique à an. Ce qujustement

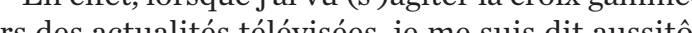

\section{西}

Réservez

votre date !

À linitiative du CIRCE (Centre Interdisciplinaire de Recherches de Paris-Sorbonne et l'Association Adice

Présentation de la revue

Témoigner entre histoire et mémoire, par Luba Jurgenson

En présence de Philippe Mesnard, directeur de la rédaction, lors de la séance des Palabres du 7 octobre 2014 à 19 h 00, à l'l'nstitut hongrois

(92, rue Bonaparte, 75006 Paris).

Organisation : Aurélie Rouget-Garma, Université Paris-Sorbonne et CIRCE.
Aurelie.Rouget-Garma@paris-sorbonne.fr

Coordination : Malgorzata Smorag-Goldberg Université Paris-Sorbonne et CIRC

maougocha@usa.net

www.facebook.com/Palabres

quelle était partout, cette croix. On ne cesse de la voi eule ou avec le portrait d'Hitler. Aussi n'est-ce pas de question de l'holocauste dans la culture qu'il faut seulement débattre, mais du nazisme dans cette mêm culture. Le plus inquiétant, c'est la naturalisation de implo enait déjà tout pour devenir une ssable

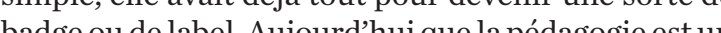
badge ou de label. Aujourd hui que la pédagogie est un

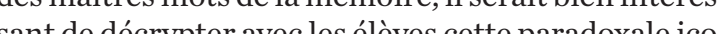
lâtrie Que pensez-vous de cette idée?

Pour en savoir plus sur Pultranationalisme ukrainien, ses nostalgies et
son soutien étatique : Iarticle particulièrement éclairant de Delphine son soutien étatique : l'article particulièremente éclairant de Delphine
Bechthel, « Les pogroms en Galicie, 1941 : des pages blanches de lhistoire

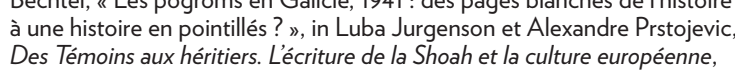
Des Témoins aux héritiers. L'écriture
Paris, éditions Petra, 2012, p. 113-135. 